Bull. Austral. Math. Soc.

VOL. 37 (1988) [165-171]

\title{
ON THE GENERALISED DOMINATED CONVERGENCE THEOREM
}

\author{
Chew Tuan Seng
}

\begin{abstract}
In this paper, we give another version of the generalised dominated convergence theorem, which is better than other convergence theorems for Perron integrals in the sense that it can be applied more easily.
\end{abstract}

\section{INTRODUCTION AND DEFINITIONS}

Recently, some convergence theorems have been proved for Perron, Denjoy and Henstock-Kurzweil integrals, namely the controlled convergence theorem $[2,3,6,7]$, the generalised mean convergence theorem [5], and the generalised dominated convergence theorem [5]. For brevity, we denote the various convergence in the above convergence theorems by CC, GMC and GDC, respectively. In [5], we proved that GDC implies CC [5, Lemma] which in turn implies GMC [6, Lemma]. Conversely, if a sequence $\left\{f_{n}\right\}$ is GMC then there exists a subsequence $\left\{f_{n(i)}\right\}$ of $\left\{f_{n}\right\}$, which is $C C[5, p .139]$. In this note, another version of the generalised dominated convergence theorem will be given, which has useful applications, as we shall see in Theorem 4 . We also prove, that if a sequence $\left\{f_{n}\right\}$ is $\mathrm{CC}$ then there exists a subsequence which is GDC.

A function $\mathrm{H}$ is said to be a major function of a function $f$ in $[a, b]$ if $\underline{D} H(x)>-\infty$ for all but a countable number of points $x$, and

$$
\underline{D} H(x) \geqslant f(x) \quad \text { almost everywhere in }[a, b]
$$

where $\underline{D}$ denotes the lower derivative. A function $G$ is said to be a minor function of $f$ in $[a, b]$ if $-G$ is a major function of $-f$ in $[a, b]$. We assume in $[5]$ that $\underline{D} H(x)>-\infty$ for every $x$ and $\underline{D} H(x) \geqslant f(x)$ for every $x$. However, for the present case, a major function $H$ is still $V B G_{\star}[8, \mathrm{p} .234]$, and furthermore the characteristic function of a set of measure zero is Perron integrable and its integral is zero. Hence it is easy to check that the generalised dominated convergence theorem [5, Theorem 2] still holds. We shall consider the following conditions:

(i) $f_{n}(x) \rightarrow f(x)$ almost everywhere in $[a, b]$ as $n \rightarrow \infty$ where each $f_{n}$ is Perron integrable on $[a, b]$ (for definitions, see $[5,8]$ );

Received 27 March 1987

Copyright Clearance Centre, Inc. Serial-fee code: 0004-9729/88 $\$ A 2.00+0.00$. 
(ii) the primitives $F_{n}$ of $f_{n}$ converge uniformly on $[a, b]$;

(ii) $t$ the primitives $F_{n}(x)$ of $f_{n}$ converge pointwise at each $x$ to a continuous function $F(x)$ in $[a, b]$;

(iii) $f_{n}$ has at least one common major function and at least one common minor function in $[a, b]$;

(iii)! $\quad[a, b]$ is the union of a sequence of closed sets $X_{i}$ and on each $X_{i}, f_{n}$ is dominated by a Lebesgue integrable function;

(iv) the primitives $F_{n}$ of $f_{n}$ are $A C G_{\star}$ uniformly in $n$ (for definitions, see $[\mathbf{5}, 8])$;

(v) $[a, b]$ is the union of a sequence of closed sets $X_{i}$ and for every $i$ and $\varepsilon>0$ there is an integer $N$ such that for every partial division of $[a, b]$ given by

$$
a_{0} \leqslant a_{1}<b_{1} \leqslant a_{2}<b_{2} \leqslant \ldots \leqslant a_{p}<b_{p} \leqslant b
$$

with $a_{1}, b_{1}, a_{2}, b_{2}, \ldots, a_{p}, b_{p}$ belonging to $X_{i}$, we have

$$
\sum_{i=1}^{p} \omega\left(F_{n}-F_{m} ;\left[a_{i}, b_{i}\right]\right)<\varepsilon \text { whenever } n, m \geqslant N
$$

where $\omega$ denotes the oscillation of $F_{n}-F_{m}$ over $\left[a_{i}, b_{i}\right]$.

We recall that $\left\{f_{n}\right\}$ is $\mathrm{CC}$ if (i), (ii) and (iv) hold, $\left\{f_{n}\right\}$ is GDC if (i), (ii) and (iii) hold and that $\left\{f_{n}\right\}$ is GMC if (i), (ii) and (v) hold.

\section{MaIN Results}

We state the main results as follows:

Theorem 1. (Second Generalised Dominated Convergence Theorem) If condition (i), (ii) and (iii)' hold, then $f$ is Perron integrable on $[a, b]$ and we have

$$
\int_{a}^{b} f_{n}(x) d x \longrightarrow \int_{a}^{b} f(x) d x \text { as } n \longrightarrow \infty .
$$

This convergence theorem can be applied more easily than other convergence theorems mentioned above, since condition (iii)', which is in terms of Lebesgue integrable functions, can easily be checked, whereas major functions in (iii) and $A C G_{\star}$ in (iv) are harder to deal with.

THEOREM 2. The above theorem holds true with condition (ii) replaced by condition (ii)'.

THEOREM 3. If a sequence of functions $\left\{f_{n}\right\}$ is $C C$ then there exists a subsequence of $\left\{f_{n}\right\}$ which satisfies (i), (ii) and (iii)'.

We note that Theorem 3 reduces to the corresponding well-known result for Lebesgue integrable functions when $X_{i}=[a, b]$ for all $i$. 


\section{Proofs of the MAIN RESUlts}

We need the following four lemmas. The first two lemmas are given in [8, p.216]. However they are modified slightly here for our purpose.

LEMMA 1. $[8$, Lemmas $2.1, p .216]$. If $g$ is a nonnegative function Lebesgue integrable on an interval $[a, b]$, then for every $\varepsilon>0$, there exists a function $G$ with $\underline{D} G(x)>-\infty$ for all $x$ except a countable number of points such that (a) $G^{\prime}(x)=g(x)$ almost everywhere on $[a, b],(b)|G(x)| \leqslant \varepsilon$ at every point $x$ of $[a, b]$.

ProOF: Let $H$ be the primitive of $g$. Let $a=a_{o}<a_{1}<a_{2}<\ldots a_{n}=b$ be a partition of $[a, b]$ such that the oscillation of $H$ is less than $\varepsilon$ on each of the intervals $\left[a_{i}, a_{i+1}\right]$, where $i=0,1,2 \ldots, n-1$. We may choose $a_{i}$ such that $\underline{D} H\left(a_{i}\right)>-\infty$ for $i=1,2 \ldots n-1$. Let $F$ be a function which is constant on each $\left[a_{i}, a_{i+1}\right]$ with $F\left(a_{i}\right)=H\left(a_{i}\right)$. Writing $G=H-F$, note that $G$ is nondecreasing on each $\left[a_{i}, a_{i+1}\right)$, thus $\underline{D} G(x)=-\infty$ only at points $x=a_{i}$, for $i=1,2 \ldots, n-1$. Furthermore, (a) and (b) hold.

Lemma 2. [8, Lemma 2.2, p.216]. If $g$ is a nonnegative function which is Lebesgue integrable on an interval $J=[a, b]$ and if $P$ is a closed set in $J$, then for every $\varepsilon>0$, there exists a function $G$ with $\underline{D} G(x)>-\infty$ for all $x$ except a countable number of points such that (a) $G^{\prime}(x)=g(x)$ at almost all the points $x$ of $J-P$, (b) $G(x)=0$ and $G^{\prime}(x)=0$ at all points $x$ of $P$ and $(c)|G(x+h)| \leqslant \varepsilon|h|$ for every $x$ of $P$ and every $h$.

Proof: This lemma follows from Lemma 1 and the proof of Lemma 2.2 in [8].

LEMma 3. Let $[a, b]$ be the union of a sequence of closed sets $X_{i}$ with $X_{i} \subseteq X_{i+1}$ for all $i$. Let $g$ be a function defined on $[a, b]$ such that $g$ is Lebesgue integrable on each $X_{i}$. Then $|g|$ is dominated by a major function.

Proof: For each $i$, define $g_{i}(x)=|g(x)|$ if $x \in X_{i}$ and zero otherwise. Then each $g_{i}$ is Lebesgue integrable on $[a, b]$. Let $G_{1}$ be the primitive function of $g_{1}$. Next we shall use Lemma 2 to define $G_{1}$ for $i \geqslant 2$. Applying Lemma 2 with $\varepsilon=2^{-i}$, $P=X_{i-1}$ and $g=g_{i}$, we obtain a function $G_{i}$ with $\underline{D} G_{i}(x)>-\infty$ for all $x$ except a countable number of points. Furthermore, we have:

(a) $\quad G_{i}^{\prime}(x)=|g(x)|$ almost everywhere in $X_{i}-X_{i-1}$, and $G_{i}^{\prime}(x)=0$ almost everywhere in $[a, b]-X_{i}$;

(b) $G_{i}(x)=G_{i}^{\prime}(x)=0$ for all $x \in X_{i-1}$;

(c) $\left|G_{i}(x+h)\right| \leqslant|h| 2^{-i}$ for all $x \in X_{i-1}$.

Let

$$
F(x)=\sum_{i=1}^{\infty} G_{i}(x)
$$


then $F$ is a finite-valued function because of (b) and (c). Let $x \in[a, b]$ and assume $x \in X_{n}-X_{n-1}$ for some $n$. Then we have

$$
\frac{F(x+h)-F(x)}{h}=\sum_{i=1}^{n} \frac{G_{i}(x+h)-G_{i}(x)}{h}+\sum_{i=n+1}^{\infty} \frac{G_{i}(x+h)-G_{i}(x)}{h} \ldots(*) .
$$

Thus

$$
\underline{D} F(x) \geqslant|g(x)|+\liminf _{h \rightarrow 0}\left[\sum_{i=n+1}^{\infty} \frac{G_{i}(x+h)}{h}\right] \text { a.e. }
$$

Consequently, in view of $(c)$, we have

$$
|g(x)| \leqslant \underline{D} F(x)+1 \leqslant \underline{D}\left[F(x)+F_{1}(x)\right] \text { a.e., }
$$

where $F_{1}(x)=x$. Note that $\underline{D} F(x)>-\infty$ for all $x$ except a countable number of points, in virtue of equality $(*)$ and condition (c). The proof is complete.

A function $F$ is said to be of bounded variation in the restricted sense on a closed set $X$ or, in short, $V B_{\star}(X)$ if $V_{\star}(F ; X)$ is finite, where

$$
V_{\star}(F ; X)=\sup \sum_{k} \omega\left(F ; I_{k}\right)
$$

and $\left\{I_{k}\right\}$ is a sequence of non-overlapping intervals whose end-points belong to $X$, $\omega\left(F ; I_{k}\right)$ denotes the oscillation of $F$ on the interval $I_{k}$ and the supremum is taken over all such $\left\{I_{k}\right\}$.

A function $F$ is said to be of generalised bounded variation in the restricted sense on $[a, b]$ or $V B G_{\star}$ if $[a, b]$ is the union of a sequence of closed sets $X_{i}$ such that on each $X_{i}$ the function $F$ is $V B_{\star}\left(X_{i}\right)$.

Lemma 4. Conditions (iii) and (iii)' are equivalent.

Proof: It follows from Lemma 3 that (iii)' implies (iii). For the converse, note that if $\underline{D} H(x)>-\infty$ for all $x$ except a countable number of points, then $H$ is $V B G_{\text {* }}$ $\left[8\right.$, Theorem 10.1, p.234]. Hence $H$ is $V B_{\star}\left(X_{i}\right)$ for all $i$, where $[a, b]=\bigcup_{i} X_{i}$ and each $X_{i}$ is closed. Consequently $H^{\prime}(x)$ exists almost everywhere and $H^{\prime}(x)$ is Lebesgue integrable on each $X_{i}$. With this fact, we see at once that (iii) implies (iii)'.

Proof of Theorem 1: This follows from Lemma 4 and the first GDC Theorem [5, Theorem 2].

Proof of Theorem 2: Theorem 2 can be deduced from Theorem 1 by using the same argument as in the proof of Theorem 2 in [7]. 
Proof of Theorem 3: We note that CC implies GMC [6, Lemma] Following the first half of the proof of Theorem 4 in [5], we obtain a subsequence $\left\{f_{n(i)}\right\}$ of $\left\{f_{n}\right\}$ which is dominated by a Lebesgue integrable function on $X_{1}$. Repeat the above process for $X_{2}$ and the sequence of primitives $F_{n(i)}$ of $f_{n(i)}$ obtained above and so on. Consequently, by the diagonal process, we obtain the required result.

\section{AN Application}

Let $D$ be the space of all Perron integrable functions defined on $[a, b]$. A functional $G$ defined on $D$ is said to be control-continuous if $G\left(f_{n}\right) \rightarrow G(f)$ as $n \rightarrow \infty$ whenever $f_{n}$ is control-convergent to $f$ in $D$. A functional $G$ defined on $D$ is said to be normcontinuous if $G\left(f_{n}\right) \rightarrow G(f)$ as $n \rightarrow \infty$ whenever $\left\|f_{n}-f\right\| \rightarrow 0$ as $n \rightarrow \infty$. Here \|\| denotes the usual norm of the space $D$, that is,

$$
\|f\|=\sup \left\{\int_{a}^{x} f(t) d t ; x \in[a, b]\right\} .
$$

It is well-known $[\mathbf{1}, 9]$ that a linear functional is norm-continuous if and only if there exists a function $g$ defined on $[a, b]$ such that $f g \in D$ whenever $f \in D$ and

$$
G(f)=\int_{a}^{b} f(t) g(t) d t .
$$

Let $L_{1}$ be the space of all Lebesgue integrable functions defined on $[a, b]$ with the usual norm \|\|$_{1}$. A functional $G$ defined on $L_{1}$ is said to be \|\|$_{1}$-continuous if $G\left(f_{n}\right) \rightarrow G(f)$ as $n \rightarrow \infty$ whenever $\left\|f_{n}-f\right\|_{1} \rightarrow 0$ as $n \rightarrow \infty$.

Theorem 4. A linear functional is norm-continuous in $D$ if and only if it is control-continuous in $D$.

Proof: The necessity follows from the fact that controlled convergence implies norm convergence. Now we shall prove the sufficiency. Let $G$ be a control-continuous linear functional in $D$. First, note that if $\left\|f_{n}-f\right\|_{1} \rightarrow 0$ as $n \rightarrow \infty$ in $L_{1}$ then for every subsequence of $\left\{f_{n}\right\}$, there exists a subsequence which is dominated by a function in $L_{1}$ and converges pointwise to $f$ almost everywhere in $[a, b]$. Thus $G$ is \|\|$_{1}$-continuous in $L_{1}$. Consequently, there exists a bounded function $g$ defined on $[a, b]$ such that

$$
G(f)=\int_{a}^{b} f(t) g(t) d t \text { for all } f \in L_{1}
$$

We shall prove that the above equality holds for all $f \in D$ by using Theorem 2 . For every $f \in D$, there exists a sequence $\left\{f_{n}\right\}$ of step functions, which is controlconvergent to $f$ (see [4]) that is to say, $\left\{f_{n}\right\}$ satisfies (i), (ii) and (iv). Obviously, 
$\left\{f_{n} g\right\}$ satisfies (i). In view of the boundedness of $g$ and the condition (iv) of $\left\{f_{n}\right\}$, $\left\{f_{n} g\right\}$ satisfies (iii)'. Next, we shall prove that $\left\{f_{n} g\right\}$ satisfies (ii)'. Note that

$$
G\left(f_{n} g \chi_{[a, x]}\right)=\int_{a}^{x} f_{n}(t) g(t) d t
$$

and, in view of the controlled continuity of $G$ and the controlled convergence of $\left\{f_{n} \chi_{[a, x]}\right\}$, we have

$$
\int_{a}^{x} f_{n}(t) g(t) d t \rightarrow G\left(f \chi_{[a, x]}\right) \text { as } n \rightarrow \infty
$$

for every $x \in[a, b]$, where $\chi_{[a, x]}$ denotes the characteristic function of an interval $[a, x]$. Thus we need to prove that for every $f \in D, G\left(f \chi_{[a, x]}\right)$ is continuous in $x$. Let $x_{n} \in[a, b]$, for $n=1,2 \ldots$, and $x_{n} \rightarrow x$ as $n \rightarrow \infty$. Let $h_{n}=f \chi_{\left[a, x_{n}\right]}$ and $h=f \chi_{[a, x]}$. Obviously, $\left\{h_{n}\right\}$ satisfies (i) and (iv). Furthermore $\left\{h_{n}\right\}$ satisfies (ii), in view of the uniform continuity of the primitive $F$ of $f$ over $[a, b]$ and the following inequality

$$
\left|\int_{a}^{y} h_{n}(t) d t-\int_{a}^{y} h(t) d t\right| \leqslant \omega\left(F ; I_{n}\right),
$$

where $\omega$ denotes the oscillation of $F$ over $I_{n}$ and $I_{n}$ is the interval with endpoints $x_{n}$ and $x$. Consequently

$$
G\left(h_{n}\right) \rightarrow G(h) \text { as } n \rightarrow \infty
$$

Thus $G\left(f \chi_{[a, x]}\right)$ is continuous in $x$. Hence, by Theorem 2,

$$
\int_{a}^{b} f_{n}(t) g(t) d t \rightarrow \int_{a}^{b} f(t) g(t) d t \text { as } n \rightarrow \infty
$$

Therefore

$$
G(f)=\int_{a}^{b} f(t) g(t) d t \text { for all } f \in D
$$

Consequently, $G$ is norm-continuous $[1,9]$.

\section{References}

[1] A. Alexiewicz, 'Linear functionals on Denjoy-integrable functions', Colloq. Math. 1 (1948), 289-293.

[2] A.G. Djvarsheishvili, 'On a sequence of integrals in the sense of Denjoy', Akad. Nauk Gruzin. SSR Trudy Mat. Inst. Rajmadze 18 (1951), 221-236.

[3] Lee Peng Yee and Chew Tuan Seng, 'A better convergence theorem for Henstock integrals', Bull London Math. Soc. 17 (1985), 557-564. 
[4] Lee Peng Yee and Chew Tuan Seng, 'A Riesz-type definition of the Denjoy integral', Real Anal. Exchange 11 (1985/86), 221-227.

[5] Lee Peng Yee and Chew Tuan Seng, 'On Convergence theorems for the nonabsolute integrals', Bull. Austral. Math. Soc. 34 (1986), 133-140.

[6] Lee Peng Yee and Chew Tuan Seng, 'A short proof of the controlled convergence theorem for Henstock integrals', Bull. London Math. Soc. 19 (1987), 60-62.

[7] Liao Ke-cheng, 'A refinement of the controlled convergence theorem for Henstock integrals', South East Asian Bull. Math. 11 (1987). No 1. .

[8] S. Saks, 'Theory of the integral'.

[9] W.L. Sargent, 'On linear functions in spaces of conditionally integrable functions', Quart. J. Math. Oxford Ser. (2) 1 (1950), 288-298.

National University of Singapore

Department of Mathematics

Lower Kent Ridge Road

Singapore 0511

Republic of Singapore 